\title{
Design and Development of Handheld Solar Powered Air Quality Measurement using LoRa
}

\author{
Manoj Kumar \\ Department of Electronics and Communication, \\ Punjab Engineering College, \\ Sector 12 Chandigarh
}

\begin{abstract}
This paper discusses design and development of portable solar powered air quality measurement device using LoRa technology. It uses RTC and GPS which allows the system to identify when and where the data is been recorded. In this paper, main objective was to focus on making system selfpowering, along with displaying the measured quantities on the sensor node and sending to the receiver node. This work also focused on making a system which can measure indoor and outdoor AQI as well. It has been tested and verified with the data measured by "aqicn.org". It is a low power consumption device which can also be powered by a standard USB. The device is able to transmit the data up to $7 \mathrm{Kms}$ with the RSSI of $-128 \mathrm{dBm}$ in urban areas and can be deployed for measurement of AQI. The device can also be used as mobile AQI measurement device, as it is fitted with GPS and RTC through which it can determine when and where the AQI is good or bad.
\end{abstract}

\section{General Terms}

Long Range, Self-powered, Sensor, Air Quality Index

\section{Keywords}

LoRa, Air Quality, WSN, Sensor Node, Receiver Node

\section{INTRODUCTION}

Air quality index tells about how polluted or clean air is, along with its effects which may cause us various health problems like sneezing, sore throat, skin burning, breathing problems, cause diseases allergies and even death to humans[1]. As per report of WHO 7 million people die every year due to air pollution[2].PM2.5 can cause severe health problems like asthma, adverse birth outcomes, decline in lung function and other cardiovascular diseases[3]. Other pollutants include $\mathrm{CO}, \mathrm{NO} 2$, SO2, VOCs, PM10, oxidants and ozone[4]. A survey by Yi et al. is presented on WSN based air quality measurement sensors and network type describe about various systems for air pollution monitoring using WSN and found WSN to be suitable for measurement of air quality[5].

LoRa modem provides Ultra long range with high interference immunity having current consumption of $2 \mu \mathrm{A}$ at sleep mode and $100 \mu \mathrm{A}$ while transmitting[6]. LoRa can be used for IOT applications on two AA cells for 6 years[7]. Lora is able to transmit up 122KM line of sight[8-9], LoRa provides secure device to device link[10] and it is also suitable for last mile Communications[11]. In a comparative study of various LPWAN Technologies it is found that LoRa is good for application where latency, quality of service, payload length is not a concern. It provides seamless range, coverage, great battery life, cost efficiency and easy deployment[12]. LoRa has found its applications in smart cities, environmental

\author{
Neelu Jain, PhD \\ Department of Electronics and Communication, \\ Punjab Engineering College, \\ Sector 12 Chandigarh
}

monitoring, smart Healthcare, smart farming[13], wireless body area network[14], wind speed estimation[15], anomaly detection in water network[16] and vibration monitoring device[17]. In paper presented by Botero-Valencia et al. on environmental monitoring using LoRa system measures various quantities such as PM2.5, PM10, O2, O3, CO2, SO2, $\mathrm{NO} 2$, temperature, $\mathrm{RH}, \mathrm{UV}$ radiations and inertial data. The system measures outdoor air quality in real-time and sends to the central node with byte size of 50 bytes[18].

\section{METHODOLOGY}

The proposed design consists of two major block Sensor Node and Receiver node as shown in Figure 1. The Sensor node senses the measurand and sends to the microcontroller via different communication protocols such as SPI, I2C and UART communication protocols. The microcontroller then processes the received raw data from sensors and presents into the readable format. The sensors data is then stored into the SD card and also send to the central node through LoRa which is communicating to microcontroller through SPI communication Protocol. The communication between Sensor Node and Receiver Node is done by LoRa communication protocol at $433 \mathrm{MHz}$. The power source for the sensor node is rechargeable battery which is charged through the solar energy. There can be many sensor nodes which can communicate to the Receiver Node. The sensor node consists of GPS which records the location data, altitude data and time of the data recorded for the data authenticity.

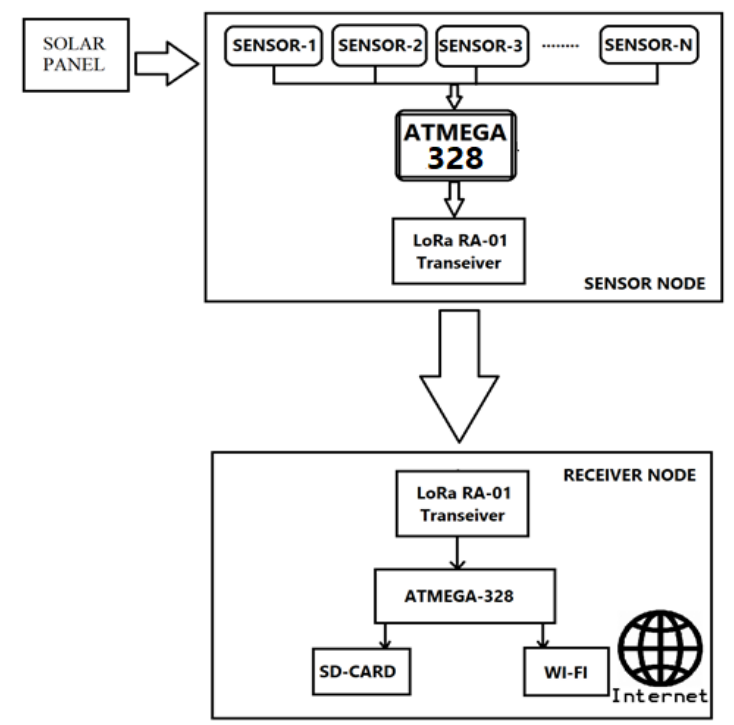

Figure 1: Block diagram of handheld solar powered air quality measurement device 
In the Receiver node the data from the sensor is received via the LoRa communication protocol. Received data from the sensor nodes is then stored in the SD card via SPI protocol for further analytics and research. The time and date of the received signal is also recorded. In order to upload the data to the cloud Wi-Fi module is been used. Received data is uploaded to the cloud via MQTT protocol.

\subsection{Sensor Node Design}

Figure 2 shows the block diagram of Sensor Node. The sensor node senses the PM2.5, PM10, temperature, humidity, pressure, $\mathrm{VOC}, \mathrm{CO} 2, \mathrm{NO} 2, \mathrm{NH} 3$, altitude and the location of the sensor through ATMEGA328 then process the data and send the data to the receiver node. All the measured quantities are stored in the SD card so that it can be viewed whenever required. The communication protocol between the sensors and the MCU is either I2C or the measurement is done through the ADC of the MCU. The raw data is processed by the MCU itself and sent to receiver node and stored in the SD card. GPS, RTC and altitude sensor play a major role in the authenticity of the data by providing the exact location of the sensor where the data is recorded by the sensor.

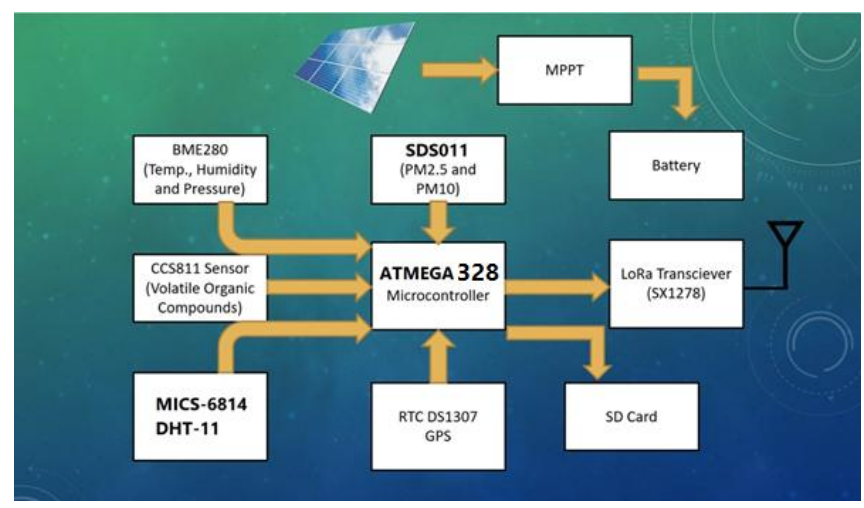

Figure 2: Block Diagram of Sensor node

\subsection{Receiver Node Design}

The receiver node is also called as central node which is situated at the central office or the data centre. The receiver node receives data from different sensor nodes and shows them in at the single location. The Receiver node consists of a LoRa Transceiver, ATMEGA328 MCU, SD card, and RTC DS1307. LoRa transceiver (based on SX1278) receives data from the sensor node and sends to the ATMEGA328 microcontroller to process the data. The data processed by MCU is saved to the SD card in the .CSV format i.e., comma separated value, so that it can be easily viewed in the Microsoft excel. The data is also send to the cloud via MQTT protocol.

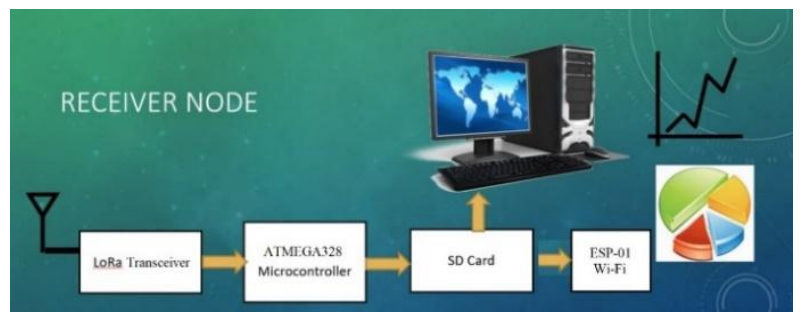

Figure 3: Block Diagram of Receiver Node

\subsection{Flow diagram of working of Sensor Node and Receiver Node}

Flow diagram of the Sensor Node is shown in figure 4. In microcontroller of the sensor node firstly all the necessary libraries are imported like "LoRa.h", "CCS811.h" etc. The libraries are required for the correct initialization of all the sensors and the communication boards. Initialization and calibration of the sensors is done to start recording data from them. Then the LoRa communication module is initialized at $433 \mathrm{MHz}$ transmission frequency.

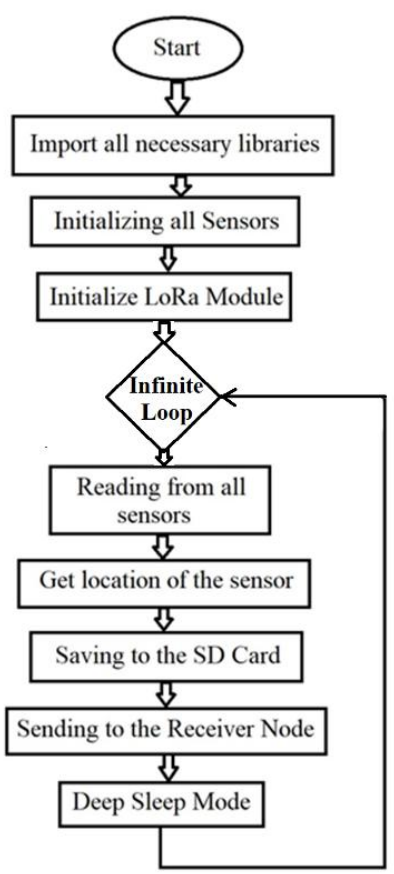

Figure 4: Flow chart of working of Sensor Node

The values of temperature, humidity, pressure, $\mathrm{NO}_{2}, \mathrm{CO}$, PM2.5 and PM10 are read from the sensors. Then all the sensor values are stored into the SD for further data interpretation. Then, sensor node sends the recorded data to the receiver node via LoRa at $433 \mathrm{MHz}$ frequency. In order to save power, the sensors and the MCU is put on deep sleep mode.

The receiver node flow diagram is shown in the figure 5 . When the receiver node is powered on MCU imports all the necessary libraries. Then the SD card, ESP-01, LoRa initialization is done at frequency of $433 \mathrm{MHz}$. There is an infinite loop which runs all the time. 


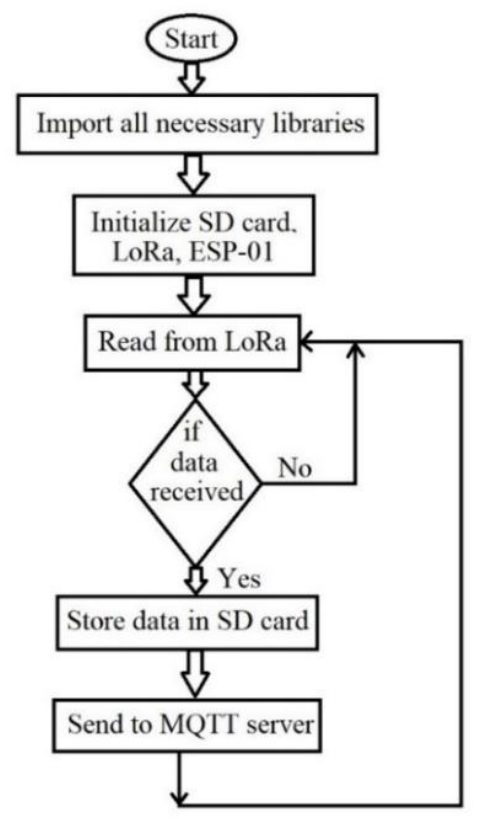

Figure 5: Flow diagram of Receiver Node

In the infinite loop if LoRa receives signal from the sensor node then the received data is stored in the SD card having file name with the "date.csv". Then the data is sent to the cloud for the real time analysis.

\section{RESULTS}

The device supports two modes "Indoor Mode" and "Outdoor Mode". The device has been tested for both the modes.

\subsection{Outdoor Mode}

The measurement setup is shown in the Figure 6 and Figure 7. By default, the sensor node enters into the "OUTDOOR MODE". The outdoor mode consumes $150 \mathrm{~mA}(750 \mathrm{~mW})$ current in Ideal mode, $200 \mathrm{~mA}(1 \mathrm{~W})$ current while sensing and sending data to the receiver Node. The device is capable of working for 24 hours only on the batteries in a single charge. In outdoor mode the sensors measure date, time, location, pressure, NO2, CO, PM2.5, PM10, Humidity and temperature.

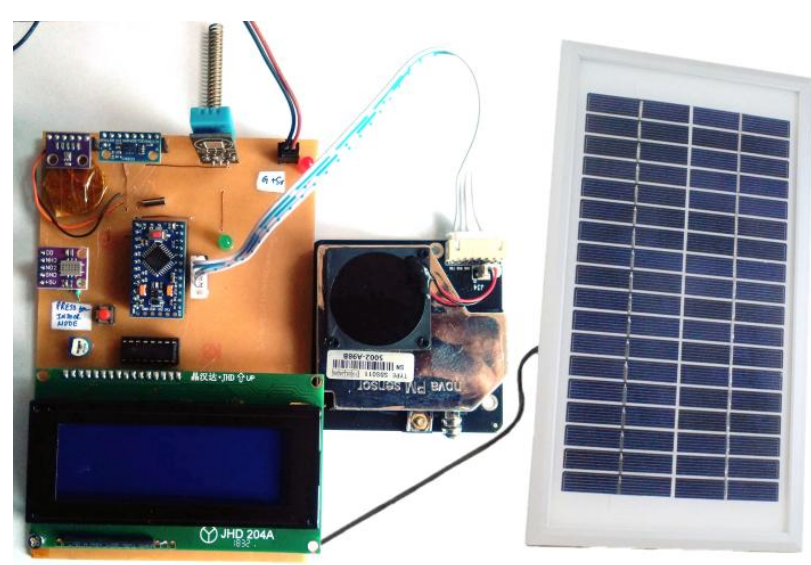

Figure 6: Measurement setup for outdoor AQI measurement

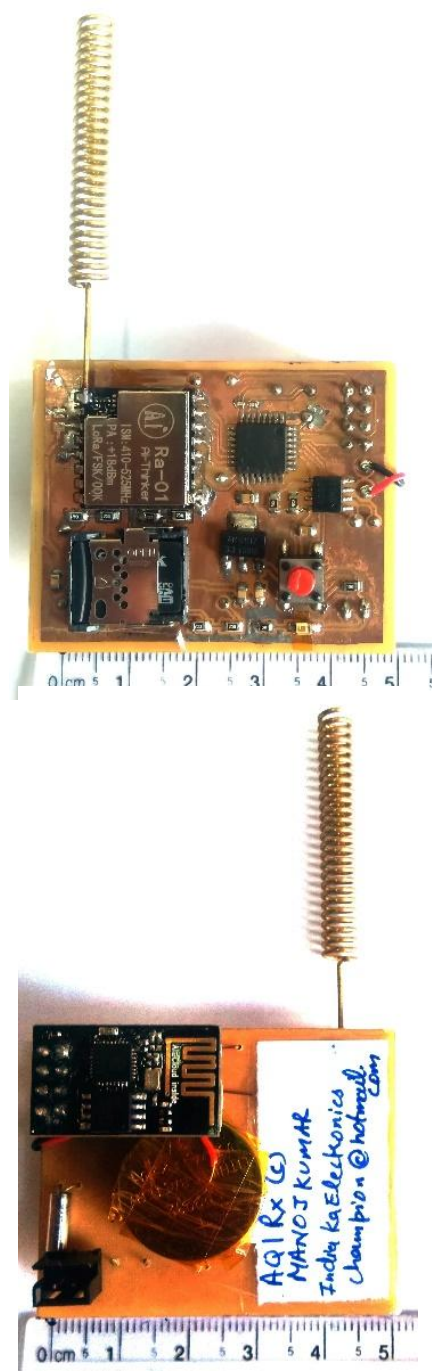

Figure 7: Assembled Receiver Node

All the measured quantities are sent over to the Receiver Node and saved and also sent over to the cloud. Following are the results obtained from the Sensor Node. The results obtained are compared with "aqicn.org" website which measures AQI in Chandigarh.

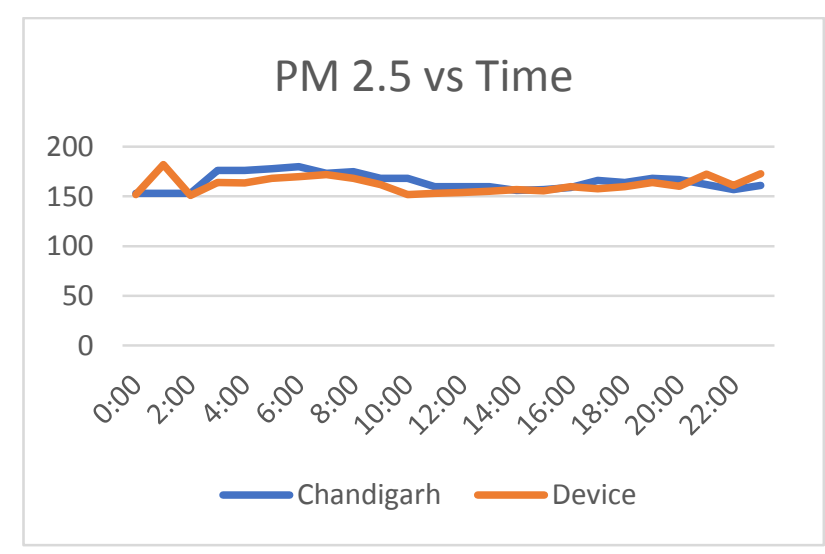

(a) PM2.5 vs time 


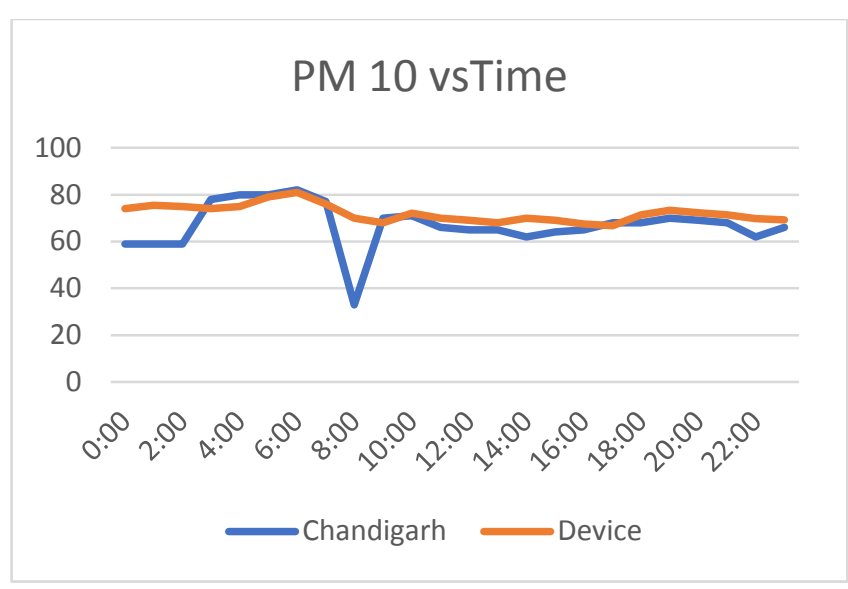

(b )PM10 vs time

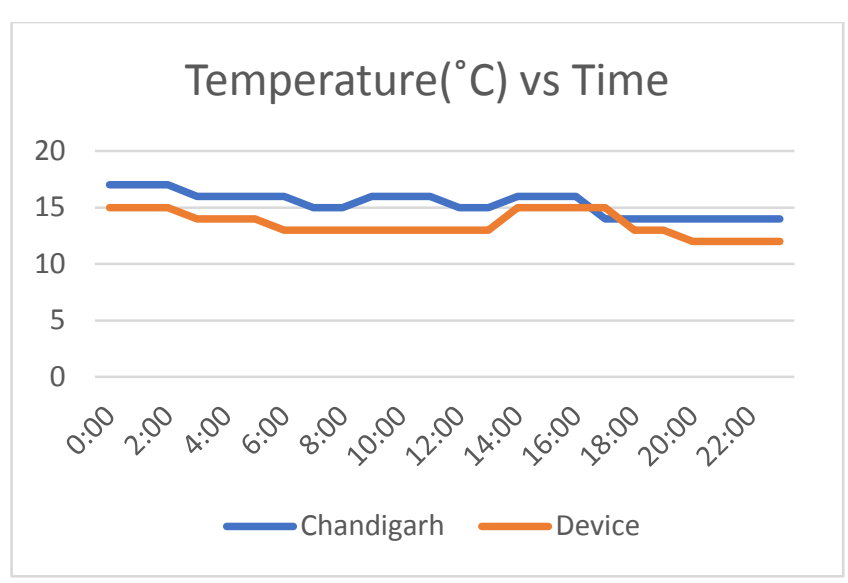

(c) Temperature vs time

\section{Humidity(\%) vs Time}

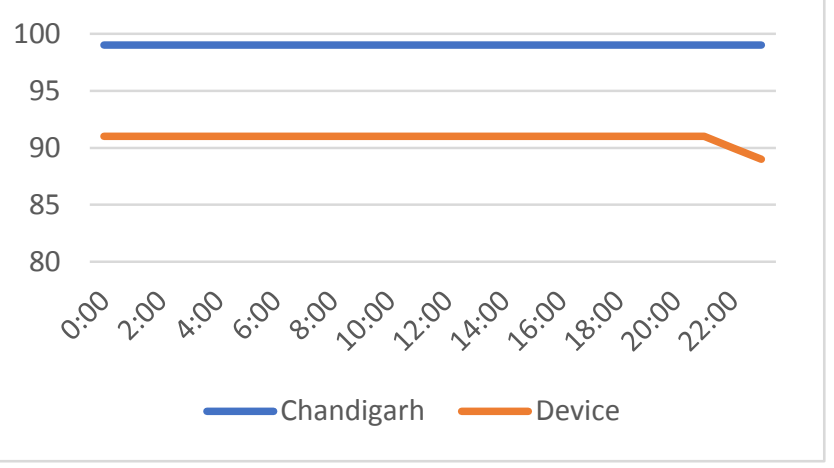

(d) Humidity vs Time

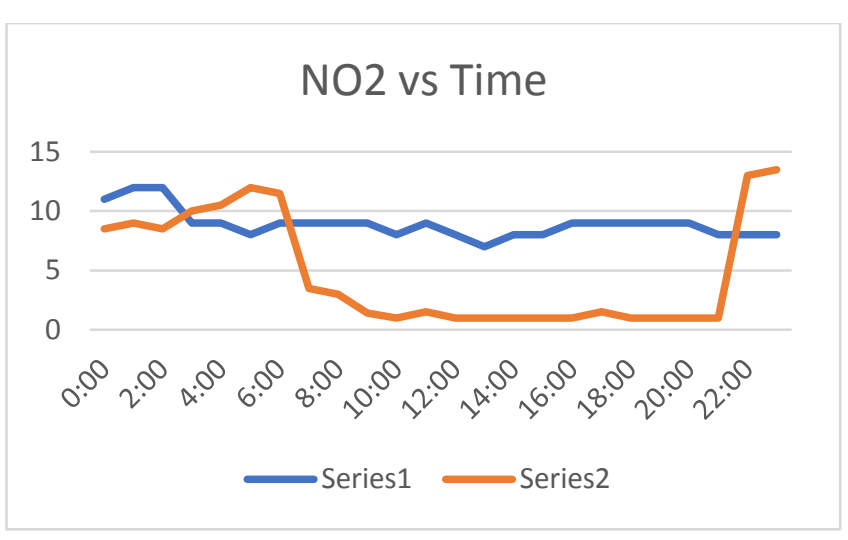

(e ) $\mathrm{NO}_{2}$ vs Time

\section{CO vs Time}

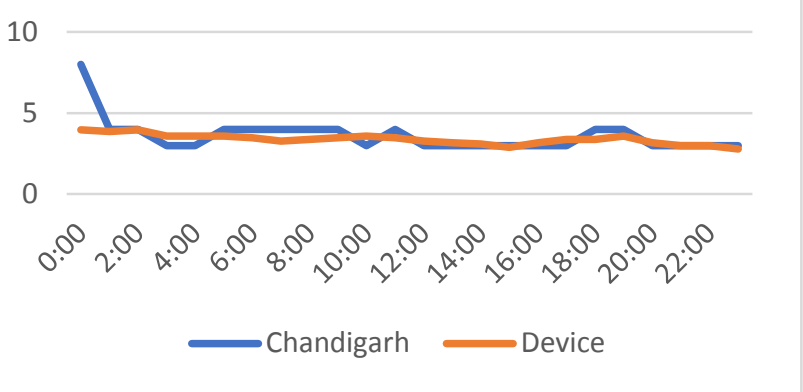

\section{(f) CO vs Time}

Figure 8: (a) PM2.5 vs Time (Hrs), (b) PM10 vs Time (Hrs), (c) $\mathrm{NO}_{2}$ vs Time (Hrs), (d) CO vs Time (Hrs), (e ) Temperature $\left({ }^{\circ} \mathrm{C}\right)$ vs Time, (Hrs) and (f) Humidity (\%) vs Time (Hrs)

Table 1: Average Air Quality Index

\begin{tabular}{|l|l|l|l|l|l|l|}
\hline & PM2.5 & PM10 & $\mathrm{NO}_{2}$ & $\mathrm{CO}$ & AQI & Category \\
\hline Prototype & 161.97 & 71.96 & 4.89 & 3.37 & 161.97 & Moderate \\
\hline Chandigarh & 164.5 & 66.91 & 8.9 & 3.600 & 164.5 & Moderate \\
\hline
\end{tabular}

The data recorded by the Sensor Node and "aqicn.org" are compared as shown in the Table 1. The AQI shows the air was moderately polluted. The location data recorded on the Sensor node is $30.767189,76.785293$, which is $20 \mathrm{~m}$ accurate. The recorded air pressure was $975 \mathrm{hPa}$. This shows that

Figure 9 shows the dashboard of cloud where all the data can be seen in real-time. The cloud dashboard shows the information of Temperature, Humidity, NO2, CO, PM10, PM2.5 and pressure. This Cloud dashboard is accessible worldwide. 


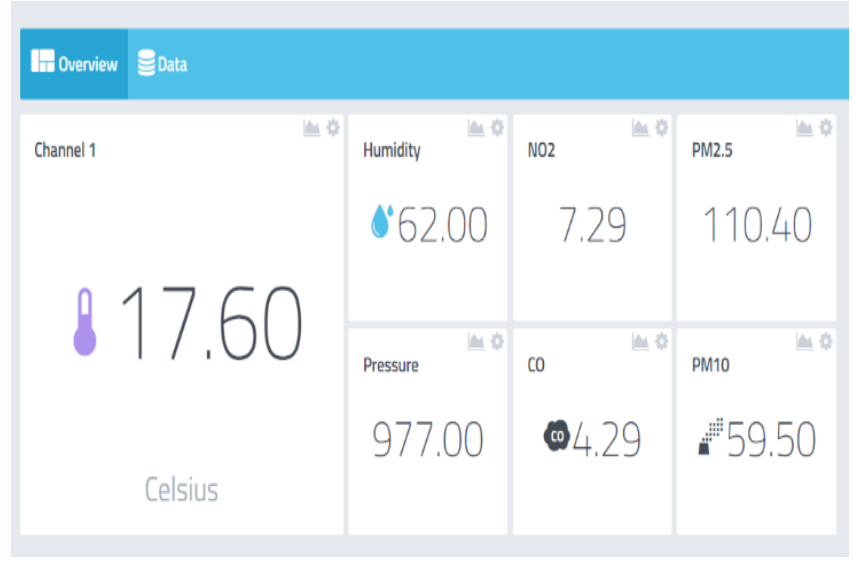

Figure 9: Cloud dashboard

\subsection{Indoor Mode}

In the Indoor mode, the Sensor Node measures the Temperature, Humidity, $\mathrm{eCO}_{2}$, TVOCs, PM2.5 and PM10. The device consumes $100 \mathrm{~mA}$ current therefore the device is capable of running in Indoor Mode for around 38-40Hrs in a single charge. All the measured quantities are shown on the LCD. To enter the Indoor Mode, Indoor Switch should be pressed for 5-10 Second when power ON or by pressing reset button of the microcontroller. In this mode the Sensor Node does not send recorded data to the Receiver node. The measurement setup is shown in the figure 10 the sensor was placed inside the LAB 2, Department of Electronics and Communication Engineering, PEC, Chandigarh.

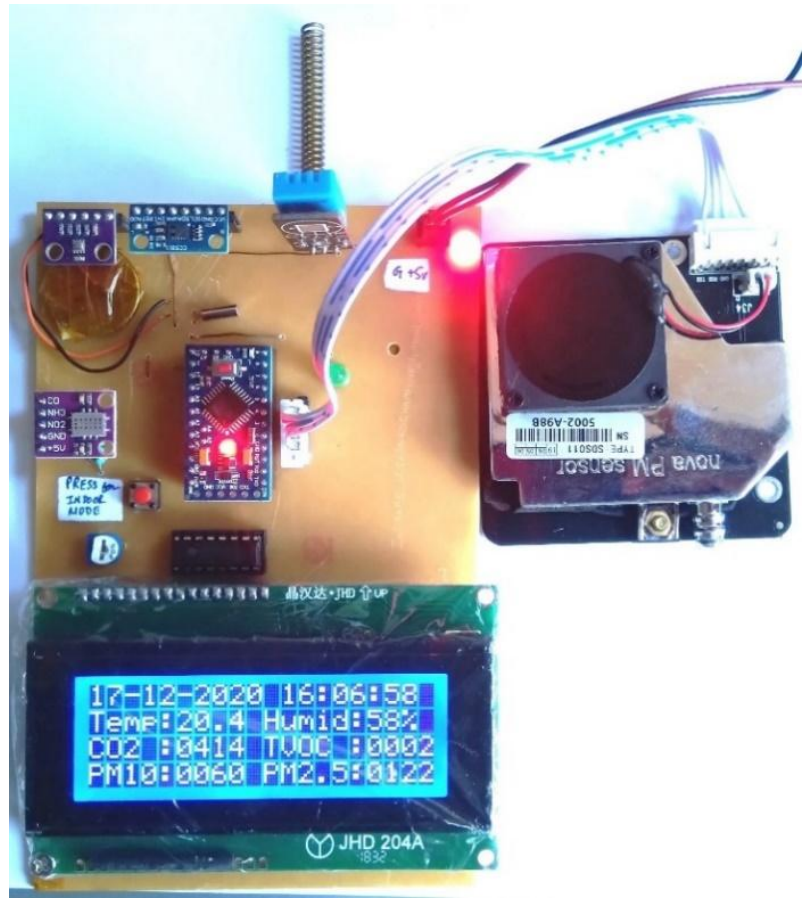

Figure 10: Measurement setup for Indoor AQI Mode

The Indoor recorded temperature and humidity is compared with the data recorded by "aqicn.org" in Chandigarh on same day as an outdoor temperature and humidity.
Table 2: Temperature and Humidity

\begin{tabular}{|l|l|l|l|l|l|l|}
\hline & \multicolumn{3}{|c|}{ Temperature } & \multicolumn{3}{c|}{ Humidity } \\
\hline & Average & Max & Min & Average & Max & Min \\
\hline Prototype & 17.32 & 18.16 & 16.78 & 69.8 & 73 & 68 \\
\hline Chandigarh & 11.38 & 17 & 9 & 86.75 & 94 & 67 \\
\hline
\end{tabular}

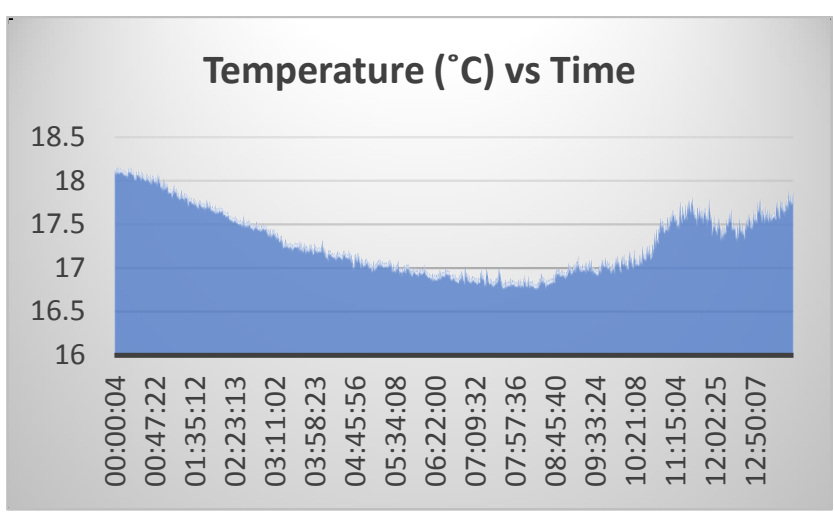

(a) Temperature $\left({ }^{\circ} \mathrm{C}\right)$ vs time

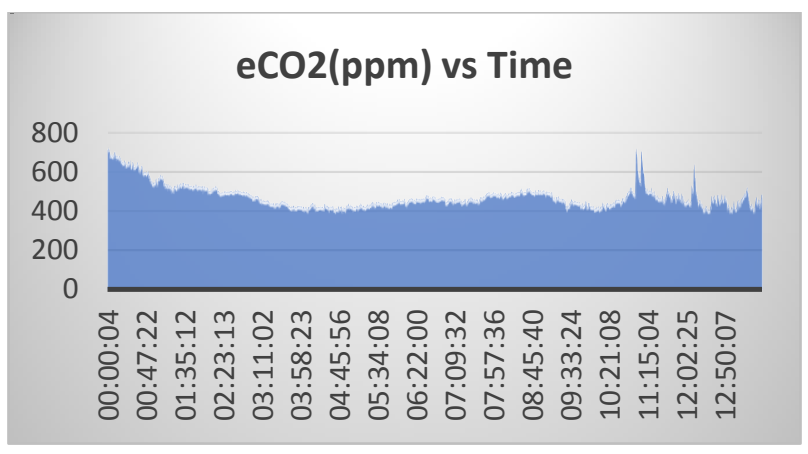

(b ) $\mathrm{eCO}_{2}$ (ppm) vs time

TVOC (ppb) vs Time

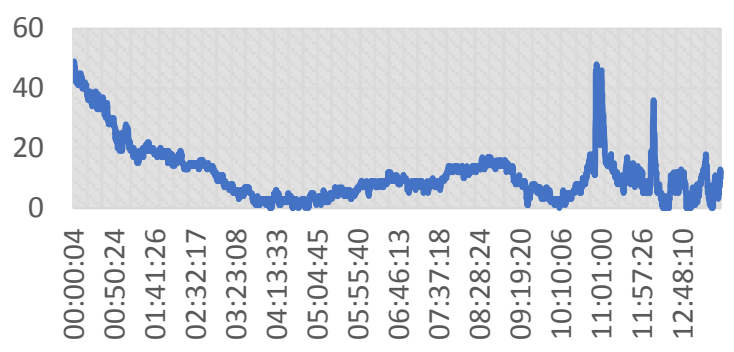

(c ) TVOCs (ppb)

Figure 11: (a) Temperature $\left({ }^{\circ} \mathrm{C}\right),(\mathrm{b}) \mathrm{eCO}_{2}(\mathrm{ppm}),(\mathrm{c})$ TVOCs (ppb) vs Time

The Indoor AQI lies the category of Moderate upon comparing from outside which lies in poor category. The indoor air quality found to be better than the outside air quality. There is increase in $\mathrm{eCO}_{2}$ and TVOCs when there are people in the room. $\mathrm{eCO}_{2}$ increases as more people in the room.

\section{CONCLUSION}

The prototype of handheld solar powered air quality measurement device was tested for both Indoor and Outdoor. 
All the measured data is sent to the receiver node with the help of LoRa modulation with range of up-to $7 \mathrm{KM}$ in the urban area with the minimum RSSI of $-128 \mathrm{~dB}$ and can cover area of $154 \mathrm{KM}^{2}$. The receiver node is able to send all the data to the MQTT server via ESP8266 which requires Wi-Fi connection. The prototype consumes very less power of maximum $1 \mathrm{~W}$ (current $200 \mathrm{~mA}$ ) and is capable of running for $24 \mathrm{Hrs}$ on a single charge.

Overall, the device is able to measure indoor and outdoor AQI and send to the receiver node and also to the cloud via MQTT protocol. The device runs on batteries and able to charge with Solar Panel.

\section{FUTURE SCOPE}

The device offers a good number of sensors, some more sensors can also be added in future to measure some more air parameters such as $\mathrm{SO}_{2}$ and $\mathrm{O}_{3}$. Sensor such as UV and luminescence could also be added to improve the device. The range of the device could also be increased by using high gain antennas and by increasing Spreading Factor and by decreasing bandwidth of LoRa.

\section{REFERENCES}

[1] Environment protection Agency, 18 June 2019, Air Quality Index (AQI) Basics, [online], Available: https://airnow.gov/index.cfm?action=aqibasics.aqi, [Accessed on 04 December 2020]

[2] World Health Organisation, 2019, How air pollution is destroying our health, [Online], Available: https://www.who.int/airpollution/news-and-events/howair-pollution-is-destroying-our-health, [Accessed 04 December 2020]

[3] Feng, S. et al. (2016) "The health effects of ambient PM 2.5 and potential mechanisms," Ecotoxicology and Environmental Safety. Academic Press, pp. 67-74. doi: 10.1016/j.ecoenv.2016.01.030.

[4] United States Environmental Protection Agency. What are the Six Common Air Pollutants? Available online: http://www.epa.gov/airquality/urbanair/ (accessed on 25 November 2020).

[5] Yi, W. Y. et al. (2015) "A survey of wireless sensor network-based air pollution monitoring systems," Sensors (Switzerland). MDPI AG, pp. 31392-31427. doi: $10.3390 / \mathrm{s} 151229859$.

[6] Semtech, 2020, Analog and mixed-signal Semiconductor | Semtech, [Online], Available: https://semtech.com/, [Accessed: 05 October 20]
[7] Bor, M., Vidler, J. and Roedig, U. (no date) LoRa for the Internet of Things. Available at: http://www.research.ibm.com/labs/zurich/ics/lrsc/lmic.ht $\mathrm{ml}$.

[8] Jovalekic, N. et al. (2018) "LoRa Transceiver with Improved Characteristics," IEEE Wireless Communications Letters, 7(6), pp. 1058-1061. doi: 10.1109/LWC.2018.2855744.

[9] Augustin, A. et al. (2016) "A study of Lora: Long range \& low power networks for the internet of things," Sensors (Switzerland), 16(9). doi: 10.3390/s16091466.

[10] Kim, J. and Song, J. (2018) “A Secure Device-To-Device Link Establishment Scheme for LoRaWAN," IEEE Sensors Journal, 18(5), pp. 2153-2160. doi: 10.1109/JSEN.2017.2789121.

[11] Bao, L. et al. (2018) "Coverage analysis on NB-IoT and LOra in power wireless private network," in Procedia Computer Science. Elsevier B.V., pp. 1032-1038. doi: 10.1016/j.procs.2018.04.252.

[12] Mekki, K. et al. (2019) "A comparative study of LPWAN technologies for large-scale IoT deployment," ICT Express, 5(1), pp. 1-7. doi: 10.1016/j.icte.2017.12.005.

[13] Zhou, Q. et al. (2018) "Design and Implementation of Open LoRa for IoT," arXiv. arXiv. doi: 10.1109/access.2019.2930243.

[14] Wu, F., Redoute, J. M. and Yuce, M. R. (2018) "WEsafe: A self-powered wearable IoT sensor network for safety applications based on lora," IEEE Access, 6, pp. 40846-40853. doi: 10.1109/ACCESS.2018.2859383.

[15] Babazadeh, M. and Karimi, K. (2019) "Development of an Arduino101-LoRa based wind speed estimator." doi: 10.1016/j.measurement.2019.06.020.

[16] Babazadeh, M. (2019) "Edge analytics for anomaly detection in water networks by an Arduino101-LoRa based WSN," ISA Transactions, 92, pp. 273-285. doi: 10.1016/j.isatra.2019.01.015.

[17] Gao, S. et al. (2019) "A multichannel low-power widearea network with high-accuracy synchronization ability for machine vibration monitoring," IEEE Internet of Things Journal, 6(3), pp. 5040-5047. doi: 10.1109/JIOT.2019.2895158.

[18] Botero-Valencia, J. et al. (2019) "Data reduction in a low-cost environmental monitoring system based on LoRa for WSN," IEEE Internet of Things Journal, 6(2), pp. 3024-3030. doi: 10.1109/JIOT.2018.2878528. 